\title{
Recreational Surfing Injuries in Cornwall, United Kingdom
}

\author{
Christopher S. M. Hay, MBBS, BSc, MRCS, FRCR; Sue Barton, MBBS; Tom Sulkin, MBBS, BSc, MRCP, FRCR \\ From the Department of Clinical Radiology, Royal Cornwall Hospital, Treliske, Truro, Cornwall, UK (Dr Hay and Dr Sulkin); and the Department \\ of Emergency Medicine, Treslisk Hospital, Truro, Cornwall, UK (Dr Barton).
}

Objective.-To analyze the nature of surfing injuries in Cornwall in order to identify trends and inform clinical management.

Methods.-The details of patients presenting (with injuries sustained while surfboard/bodyboard riding) to the Emergency Department (ED) of the Royal Cornwall Hospital, Truro (UK), from September 2004 until August 2006 were recorded prospectively. The notes were then retrospectively reviewed by a senior ED physician. The records of each visit were scrutinized for date, age, sex, injury type, and injury severity and outcome; in addition, the patient's residential status (Cornish resident or visitor) was recorded.

Results.-A total of 212 patient episodes were collected. Male patients represented $80 \%$ of injuries. The average age was 27 years (range, 11-66 years). Nonresident surfers represented 57\% (121) of the patients, and 43\% (91) of patients were local Cornwall residents. Of the total injuries, 90\% $(n=190)$ were injuries that were minor/moderate (allowing for discharge after treatment). Lacerations accounted for $38 \%(n=73)$ of injuries, and bruising and laceration to the head represented $37 \%(n=71)$ of injuries in this group. Sprains to the neck and back represented 53\% (21/40) of all sprains. Fractures of the facial bones represented 6 of the 8 fractures to the head region; the other 2 fractures involved teeth. Fractures to the upper and lower limbs were equally frequent (6 cases of each). Anterior shoulder dislocations accounted for $10 \%(n=19)$ of injuries not requiring hospital admission. Injuries requiring hospital admission represented $10 \%(n=22)$. These injuries were a more disparate group, with fractures of the cervical spine and skull accounting for 32\% $(n=7)$ and fractures of the lower limb $27 \%$ $(n=6)$ of the total. A total of $77 \%(n=168)$ of all injuries presented in the summer months (April through September). The greatest number of presentations occurred in August, with $33 \%(n=69)$ of the total surf-related injuries for the year.

Conclusions.- Surfing injuries were most common in young adult men. Most injuries presenting to the ED were minor/moderate injuries and did not require hospital admission. The overall pattern of injuries was similar to those found in studies from other countries where surfing is popular; however, there was a higher-than-expected incidence of shoulder dislocation. The trends identified in this study could be used to inform education focused on prevention of the most common injuries. Increased use of protective headwear should be considered.

Key words: surfboard, riding, injury, prevention, head, shoulder, Cornwall (UK)

\section{Introduction}

Previous research ${ }^{1,2}$ into the frequency and pattern of surfing injuries among recreational surfers in the United States and Australia have demonstrated a preponderance of lacerations, contusions, and sprains, with the head and

Corresponding author: Christopher Hay, MBBS, BSc, MRCS, FRCR, Department of Radiology, Royal Cornwall Hospital, Treliske, Truro, Cornwall, UK, TR1 3LJ (e-mail: c.hay@nhs.net). lower limbs being the most frequent anatomical sites affected. The more experienced surfer surfing on larger waves has a higher relative risk of serious injury. ${ }^{1}$ There was a preponderance of head injuries demonstrated in all studies. ${ }^{1-3}$ The use of headgear is rare among surfers; the most common reason cited by surfers is that there is "no need', for headgear. 4

In addition to traumatic injuries, overuse injuries of the neck and lower back and shoulder are frequently 
encountered among recreational surfers. ${ }^{1-5}$ Research $^{6-8}$ regarding competitive surfers reflects a similar pattern of injuries, again with lacerations predominating (again, those to the head), but sprains and strains are observed more frequently. Physiological studies ${ }^{8}$ into the performance of competitive surfers indicate a relatively high level of aerobic fitness, reflecting the demands of the competitive sport and also the continual exposure to adverse environmental factors. In the novice or untrained surfboard rider exhaustion may have implications with regard to the injury pattern.

The Royal Cornwall Hospital, Truro, houses the main emergency department (ED) in Cornwall, and therefore it sees a high proportion of the county's surfing injuries. Surfing enjoys great popularity in Cornwall.

This study was undertaken to determine the injury type and number as well as the demographics related to the recreational pursuit of surfboard/bodyboard riding in Cornwall, as reflected by those surfers presenting to a single ED.

\section{Materials and methods}

Over the period from September 2004 to August 2006 our group recorded the clinical details of patients presenting with a surfing-related injury presenting to the ED of the Royal Cornwall Hospital, Truro. Injuries sustained on short board, long boards, and bodyboards were included. Injuries sustained windsurfing, kite surfing, and skim-boarding were excluded.

The study was publicized in the department by a senior member of clinical staff (S.B.) as well as with posters displayed in staff areas (coffee lounge and changing rooms). The patient details (sticker) of any patients with a surfing-related injury were recorded in a book provided for this purpose in the clinical area; notes review was then undertaken by a senior ED physician at a later time (S.B.). The records of each ED visit were scrutinized for date, age, sex, injury type, and injury severity and outcome; in addition, the patient's residential status (Cornish resident or visitor) was recorded.

The main ED in Cornwall is at the Royal Cornwall Hospital ( $\mathrm{RCH})$ in Truro. There is a satellite unit in Penzance as well as a number of minor injury units located elsewhere in the county. Data were only collected from presentations to the Royal Cornwall Hospital.

Ethical approval was sought from the Cornwall \& Plymouth Research Ethics Committee. A formal ethics committee application was waived by the Chairman under the terms of the Governance Arrangements for Research Ethics Committees in the UK.

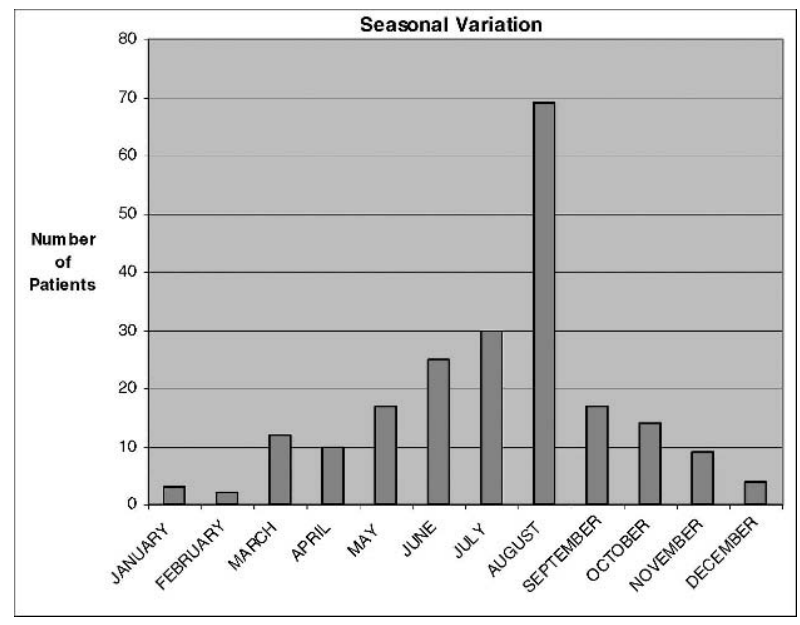

Figure. Seasonal variation.

\section{Results}

Over a 2-year period between September 2004 and August 2006, 212 patient episodes were collected. Male patients represented $80 \%$ (170 cases) of patients. The average age was 27 years, with ages ranging from 11 to 66 years. Nonresident surfers represented $57 \%$ (121) of the patients; $43 \%$ (91) of patients were local Cornwall residents. Most injuries were recorded in the summer months (April through September; 77\% $[n=168]$ ). The greatest number of presentations occurred in August, with $33 \%(n=69)$ of the total surf-related injuries for the year (the Figure).

\section{MINOR/MODERATE INJURIES}

Of the total injuries, 90\% (190 cases) were minor/ moderate or allowed for discharge after treatment (Table 1). Lacerations accounted for $38 \%$ (73 cases) of injuries, and bruising and laceration to the head represented $37 \%$ (71 cases) of injuries in this group. Sprains to the neck and back represented 53\% (21/40) of all sprains.

The miscellaneous group included patients presenting with ruptured tympanic membrane $(n=3)$, scrotal laceration $(n=1)$, and a torn medial meniscus $(n=1)$. Fractures of the facial bones represented 6 of the 8 fractures to the head region; the other 2 fractures involved teeth. Fractures to the upper and lower limbs were equally frequent ( 6 cases of each). Anterior shoulder dislocations accounted for $10 \%(n=19)$ of injuries not requiring hospital admission.

\section{SERIOUS INJURY}

Injuries requiring hospital admission represented 10\% ( $=22$ ) of injuries (Table 2). These injuries were a more 
Table 1. Minor/moderate surfing-related injuries $(n=190)$. These patients were suitable for discharge from the Accident and Emergency Department following treatment. Note the high numbers of bruising or lacerations to the head $(n=71)$ and the high number of anterior shoulder dislocations $(n=19)$

\begin{tabular}{|c|c|c|c|c|c|}
\hline Site & Bruising & Laceration & Sprain & Fracture & Dislocation \\
\hline Head & 16 & 55 & 0 & 8 & 0 \\
\hline Neck & 0 & 0 & 17 & 0 & 0 \\
\hline Back & 0 & 0 & 4 & 0 & 0 \\
\hline Chest/ribs & 5 & 0 & 0 & 2 & 0 \\
\hline Shoulder & 0 & 0 & 0 & 1 & 19 \\
\hline Upper limb & 0 & 7 & 6 & 6 & 4 \\
\hline Lower limb & 5 & 10 & 13 & 6 & 1 \\
\hline Miscellaneous & 0 & 1 & 0 & 4 & 0 \\
\hline Total & 26 & 73 & 40 & 26 & 24 \\
\hline
\end{tabular}

disparate group, with fractures of the cervical spine and skull accounting for $32 \%(n=7)$ of injuries and fractures of the lower limb accounting for $27 \%(n=6)$ of the total.

\section{Discussion}

A study of the number and type of injuries sustained while surfing in the UK has not previously been published. Previous studies into the incidence of surfboard riding injuries in recreational and professional surfers in other countries have informed important surf safety recommendations. ${ }^{1-4}$ This study demonstrated that $80 \%$ of the injuries occurred in male patients. The mean age was 27 years. This preponderance of young males is very similar to observations in other studies ${ }^{2}$ and identifies the demographic that needs to be targeted in primary prevention. Lacerations and bruising to the head

Table 2. Serious surfing-related injuries requiring hospital admission $(n=22)$. This group of injuries was more heterogeneous; many of the injuries imply high-energy transfer

\begin{tabular}{lc}
\hline \multicolumn{1}{c}{ Injury type } & No. \\
\hline Skull fracture & 4 \\
Cervical spine fracture & 3 \\
Prolapsed intervertebral disk & 1 \\
Pelvic fracture & 1 \\
Upper limb fracture & 1 \\
Shoulder dislocation & 1 \\
Lower limb fracture & 6 \\
Hip dislocation & 1 \\
Fractured larynx & 1 \\
Fractured pancreas & 1 \\
Urethral rupture & 1 \\
Drowning (fatal) & 1 \\
Total & 22 \\
\hline
\end{tabular}

represented $38 \%$ of all injuries in this study. Again, this result is in alignment with the results of other studies. ${ }^{1-8}$ It is likely that much of this injury burden could be avoided with the use of headgear, but as demonstrated in other studies, ${ }^{4}$ headgear is rarely worn by surfers. Surfboard design has taken into account the risk to others of sharp fins and points, and beginner boards used on crowded beaches by surf schools often utilize board designs that lack sharp edges and that are of softer foam construction. Extending the use of this type of board in the rental market may help reduce the incidence of trivial injuries.

There was a high incidence of anterior shoulder dislocation. This incidence is higher than previously described in the literature. These high numbers may be indicative of the number of inexperienced surfers in Cornwall, but the exact mechanism is not clear from this study. This represents an area for further research and may represent another area for focused primary prevention.

The disparate nature of injuries in the "serious injuries" group is a reflection of the potential highenergy trauma seen in surfboard riding, as documented in other studies. ${ }^{3,8}$ These injuries are seen more frequently during winter months among resident surfers. The authors propose that the larger waves seen during these months are sought out by resident surfers aiming to achieve greater "thrills," simultaneously exposing themselves to greater risk of serious injury.

Very large numbers of tourists visit Cornwall in the summer holiday season (the population of Cornwall increases by $60 \%$ in the month of August ${ }^{9}$ ). In an effort to combat the potential for injury from surf and other beach activities, the lifeguard presence on the beaches has increased over the last few years through the concerted efforts of the Royal National Lifeboat Institute and its Beach Lifeguards service. The number of surfers/ beachgoers receiving aid/rescue increased threefold in 
the years 2001 through $2004 .^{10}$ Much of their work is given over to education and prevention, a role to be encouraged, given the findings of our study. Given the rising popularity of surfing, it is likely that the incidence of surfing-related injuries will continue to rise unless efforts are made to address some of the simple preventative measures indicated in our study. Our data echo the studies from Australia and the United States indicating the demographic of surfers and their medical needs. Safety recommendations made based on these studies can therefore reasonably be used in the UK.

The data collected in this study represent cases presenting to the main ED at Truro Hospital and do not take into account the smaller local minor treatment units located elsewhere in the county. This no doubt results in some underreporting of the surfboard/bodyboard riding injuries. Also, data collection did not allow for determination of the time spent surfboard riding, which in turn does not allow us to determine the true incidence of recreational surfing injuries in this country. It would have been informative to record the level of experience and exact type of board used by the injured surfboard rider. We also recognize the potential for underreporting as a result of the voluntary nature of our data collection; we believe that this was in some way offset by the unit's enthusiasm for surf sports and for this study.

Areas for further research include the following:

1. A beach-based study to establish the true incidence of injuries pertinent to certain locations. This would assist in planning for the lifeguard service.

2. A focused investigation into the mechanism of shoulder dislocation among surfers, including analysis of type of board and level of experience, given the unusually high numbers demonstrated in this study.

\section{Conclusions}

Surfing in Britain is not without risk. This study has identified trends in surfing injuries in the UK that are very similar to the findings of previous studies from other countries where surfing is popular. We would maintain that our study can inform education focused on prevention of the most common injuries, particularly during the summer months, when these injuries are most frequent. In particular, the high prevalence of head injury is common to all studies, and we would therefore recommend that helmets should be available at all surf outlets and that their use be encouraged. The number of anterior shoulder dislocations seen in our study was higher that we would have expected, given the existing literature. Further study is required before our findings can be used to inform education and prevention of this particular type of injury. Recognition of the nature of surfboard riding injuries is the first step in making it a safer sport.

\section{References}

1. Nathanson A, Haynes P, Galanis D. Surfing injuries. Am J Emerg Med. 2002;20:155-160.

2. Taylor DM, Bennett D, Carter M, Garewal D, Finch CF. Acute injury and chronic disability resulting from surfboard riding. J Sci Med Sport. 2004;7:429-437.

3. Lowdon BJ, Pateman NA, Pitman AJ. Surfboard-riding injuries. Med J Aust. 1983;2:613-616.

4. Taylor DM, Bennett D, Carter M, Garewal D, Finch CF. Perceptions of surfboard riders regarding the need for protective headgear. Wilderness Environ Med. 2005;16:7580.

5. Hartung GH, Goebert DA, Taniguchi RM, Okamoto GA. Epidemiology of ocean sports-related injuries in Hawaii: 'Akahele O Ke Kai.' Hawaii Med J. 1990;49:52, 54-56.

6. Nathanson A, Bird S, Dao L, Tam Sing K. Competitive surfing injuries. A prospective study of surfing related injuries amongst contest surfers. Am J Sports Med. 2007; 35:113-117.

7. Lowden BJ, Pitman AJ, Pateman NA, et al. Injuries to international competitive surfboard riders. J Sports Med Phys Fitness. 1987;27:57-63.

8. Mendez-Villanueva A, Bishop D. Physiological aspects of surfboard riding performance. Sports Med. 2005;1:55-70.

9. Cornwall County Council. Tourism in Cornwall. Available at: http://db.cornwall.gov.uk/ltp/annex2/chapter-57/htm. Accessed September 20, 2008.

10. The RNLI Beach Lifeguards report 2001-2004. Page 6. Available at: www.rnli.org.UK. Accessed September 20, 2008. 